\title{
Mechanical thrombectomy and carotid artery stenting for stenosis of the internal carotid artery with free-floating thrombosis: illustrative case
}

\author{
Ryuta Tomoyose, MD, ${ }^{1,2}$ Tomoyuki Tsumoto, MD, PhD, ${ }^{1}$ Kenta Hara, MD, ${ }^{1}$ Yuichi Miyazaki, MD, ${ }^{1}$ So Tokunaga, MD, ${ }^{1}$ \\ Masahiro Yasaka, MD, $\mathrm{PhD},{ }^{2}$ and Yasushi Okada, MD, $\mathrm{PhD}^{2}$ \\ Departments of ${ }^{1}$ Neuroendovascular Surgery and ${ }^{2}$ Cerebrovascular Medicine and Neurology, Clinical Research Institute, National Hospital Organization, Kyushu Medical \\ Center, Fukuoka, Japan
}

\begin{abstract}
BACKGROUND The authors report a case in which mechanical thrombectomy and carotid artery stenting (CAS) were performed for acute cerebral infarction with free-floating thrombosis (FFT) in left internal carotid artery (ICA) stenosis. Good results were obtained.

OBSERVATIONS A 63-year-old man developed sudden disturbance of consciousness and right hemiplegia. He was transported to the authors' hospital by an emergency vehicle. Head magnetic resonance imaging showed acute cerebral infarction in the left middle cerebral artery region, and magnetic resonance angiography showed poor vascular flow beyond the left ICA. Emergency angiography revealed severe stenosis at the origin of the left ICA and a free-floating thrombus attached to the stenosis and extending to the peripheral side. Percutaneous transluminal angioplasty (PTA) was performed on the stenosis with proximal protection, the thrombus was aspirated under reversal flow, and CAS was performed without exacerbation of clinical symptoms.
\end{abstract}

LESSONS PTA, thrombus aspiration, and CAS under reversal flow may be effective treatments for FFT caused by ICA stenosis.

https://thejns.org/doi/abs/10.3171/CASE21338

KEYWORDS free-floating thrombosis; mechanical thrombectomy; carotid artery stenting

Free-floating thrombosis (FFT) is a rare cause of cerebral infarction, but it is highly likely to cause symptoms ${ }^{1}$ and requires appropriate treatment. On the one hand, there have been few reports of FFT and no clear treatment guidelines. On the other hand, the number of reports of endovascular treatment is increasing due to the progress of devices and technology. In this article, we report a case of effective treatment of cerebral infarction caused by FFT with internal carotid artery (ICA) stenosis by thrombus retrieval and carotid artery stenting (CAS) under flow reversal.

\section{Illustrative Case}

A 63-year-old male who had a history of percutaneous coronary intervention for myocardial infarction and was receiving an antiplatelet drug (clopidogrel) was living at home as usual until one morning.
At lunch, his speech suddenly became slurred, and he was unable to stand up from his chair; consequently, his wife called for emergency medical assistance, and he was rushed to our hospital. The patient's consciousness gradually improved. On the basis of findings of carotid ultrasonography and head magnetic resonance imaging (MRI), we diagnosed multiple atherothrombotic cerebral infarctions caused by artery-to-artery embolism with severe stenosis of the left ICA. Because of the rapid improvement in symptoms, intravenous tissue plasminogen activator therapy was not administered. On the same day, urgent angiography of the head was performed to evaluate severe stenosis of the left ICA, and the North American Symptomatic Carotid Endarterectomy Trial criteria showed moderate stenosis of $60 \%$ at the origin of the left ICA and the fluoroscopic image showed $3 \times 46-\mathrm{mm}$ thrombus extending from the

ABBREVIATIONS ADAPT = direct aspiration pass technique; $\mathrm{CAS}=$ carotid artery stenting; $\mathrm{CCA}=$ common carotid artery; $\mathrm{CEA}=$ carotid endarterectomy; $\mathrm{CT}=$ computed tomography; $\mathrm{ECA}=$ external carotid artery; FFT = free-floating thrombosis; $I C A=$ internal carotid artery; $\mathrm{MCA}=$ middle cerebral artery; $\mathrm{MRA}=$ magnetic resonance angiography; $\mathrm{MRI}=$ magnetic resonance imaging; $\mathrm{MT}=$ mechanical thrombectomy; $\mathrm{PTA}=$ percutaneous transluminal angioplasty; SR = stent retriever. INCLUDE WHEN CITING Published August 30, 2021; DOI: 10.3171/CASE21338

SUBMITTED June 5, 2021. ACCEPTED June 11, 2021.

(C) 2021 The authors, CC BY-NC-ND 4.0 (http://creativecommons.org/licenses/by-nc-nd/4.0/). 

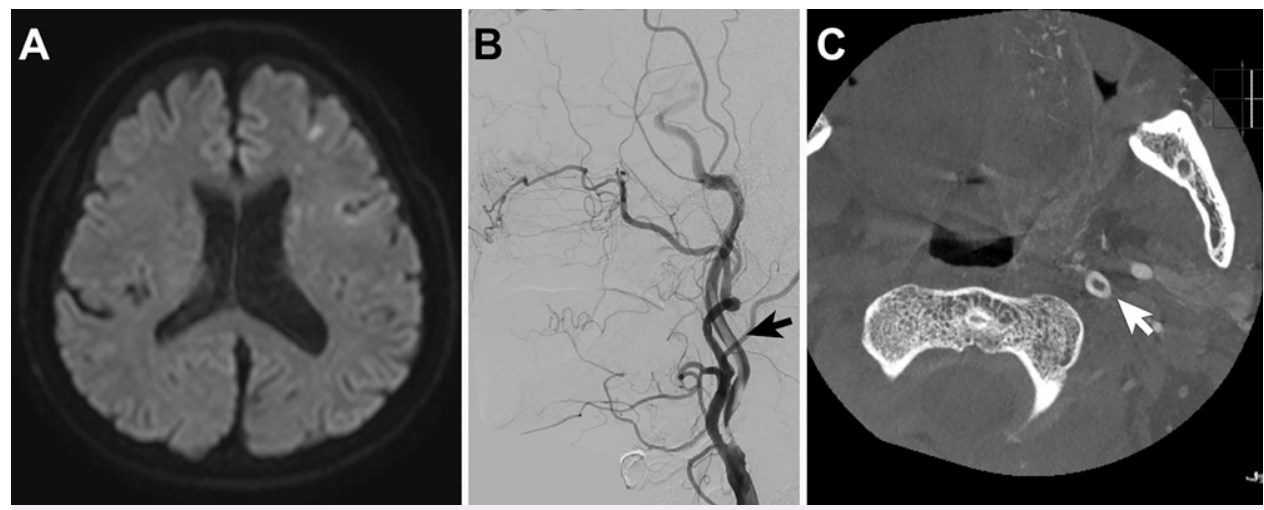

FIG. 1. A: Diffusion-weighted MRI showing a high-intensity area in the left MCA territory. B: Angiography showing elongated FFT extending along the left ICA. C: High-resolution CT in the left ICA showing donut signs.

stenosis to the peripheral side. Contrast-enhanced cone beam computed tomography revealed a donut sign in the left ICA (Fig. 1).

On the basis of the examination findings, the patient was diagnosed with ICA stenosis complicated by an FFT. We decided to perform emergency revascularization because the patient had had a stroke while receiving antiplatelet medication, and there was a high possibility that his condition would worsen due to the presence of an unstable thrombus. As a treatment strategy, we decided to block the left external carotid artery (ECA) and common carotid artery (CCA), remove the floating thrombus using a suction catheter under flow reversal, and add stenting if necessary. Aspirin $200 \mathrm{mg}$, clopidogrel $300 \mathrm{mg}$, and an argatroban infusion were started in preparation for stent placement. A 9-French long sheath was placed in the right femoral artery, a 6-French long sheath was placed in the left femoral artery, and a 9-French balloon guiding catheter (OPTIMO; Tokai Medical Products) and a 5-French guiding catheter (Envoy; J\&J Codman) were placed in the CCA. First, a 5-French Envoy catheter guided the Carotid GuardWire PS (Medtronic Vascular) into the left ECA. When the left ECA and CCA were blocked, there was no change in the neurological symptoms; it was therefore judged that there was tolerance, and treatment was continued. Next, the second GuardWire from the 9-French OPTIMO catheter was used to cross the lesion and guide it to the left ICA, and percutaneous transluminal angioplasty (PTA) was performed on the left ICA stenosis using RX-Genity (3.5 mm $\times 40 \mathrm{~mm}$; Kaneka Medics) in reversal flow to secure a vessel diameter that would allow the suction catheter to safely pass through the stenosis (10 atm, 30 seconds).

After the RX-Genity was removed and the Penumbra ACE68 catheter was guided along the axis of the GuardWire in the left ICA, the

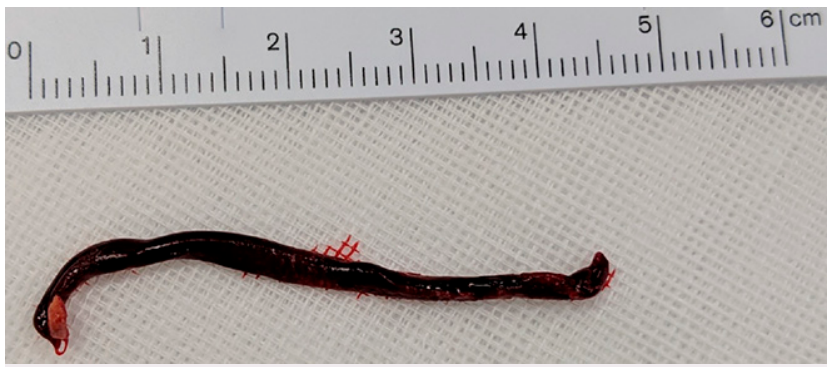

FIG. 2. The 45-mm red clot was removed from the left MCA after endovascular thrombectomy.
GuardWire implanted into the left ICA was temporarily removed to prevent a decrease in suction force. With the left ECA and left CCA blocked, the Penumbra ACE68 was connected to the suction pump and suctioned back and forth. Next, the GuardWire was guided to the left ICA again, and the Penumbra ACE68 was removed with the ICA blocked. The aspirated blood was found to contain a 40 -mm-long red thrombus (Fig. 2). After the left CCA was unblocked and switched to distal protection, retrograde angiography was performed using an export aspiration catheter attached to the GuardWire; the image of the contrast defect in the left ICA disappeared, but stenosis remained. An 8-mm $\times$ 40-mm catheter (Precise Pro Rx; Cardinal Health) was deployed into the stenotic area and confirmed to be well dilated (Fig. 3), and the procedure was completed. MRI showed a new petechial infarction in the cortex of the left middle cerebral artery (MCA) territory, but it was not possible to determine whether the infarction had occurred before endovascular treatment or whether it was a complication of the treatment procedure; nonetheless, there was no worsening of neurological symptoms. Magnetic resonance angiography (MRA) showed improved vascularization from the left ICA to the left MCA. Carotid echocardiography showed no plaque protrusion or thrombus formation in the stent of the left ICA, and the peak systolic velocity improved to $113 \mathrm{~cm} / \mathrm{second}$. The patient was discharged to home on the 11th day of the disease course without recurrence of cerebral infarction. Six months later, follow-up angiography showed no restenosis, so the antiplatelet therapy was reduced to a single agent, and there was no recurrence of cerebral infarction.

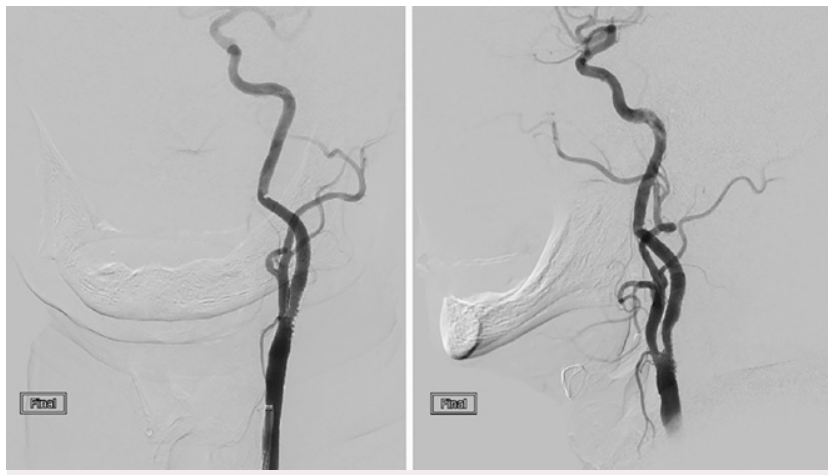

FIG. 3. Left: Frontal angiography of the left ICA showing no residual stenosis and no thrombosis. Right: Lateral view. 


\section{Discussion \\ Observations}

FFT is characterized by periodic movements associated with cardiac contraction and is defined as the presence of elongated thrombi surrounded by blood flow on the distal side. The prevalence ranges from $0.05 \%$ to $0.7 \%$ in patients with acute stroke, with a male-to-female ratio of $2: 1$ and a mean age of 57.6 years, which tends to be younger than the mean age of patients with carotid atherosclerosis (66 years). ${ }^{2}$ In addition, $92 \%$ of patients with FFT have neurological symptoms. ${ }^{1}$ Stenotic lesions due to atherosclerosis are the most common cause of thrombus formation, and changes in blood flow around the stenosis are considered a risk for thrombus formation. ${ }^{3}$ Other causes include hypercoagulable states, cardiogenic embolism, autoimmune diseases, carotid artery aneurysms, and aortic dissection. ${ }^{1}$ Computed tomography (CT) angiography, contrast-enhanced MRA, and arteriography with cerebral angiography are the gold standards for diagnosis. ${ }^{4}$ However, carotid artery ultrasound is noninvasive and safe and allows repeated observation of thrombi and stenotic lesions, making it useful for evaluation to determine a treatment strategy. ${ }^{5}$ A horizontal section of CT angiography shows a circular halo $\operatorname{sign}^{6}$ and a donut $\operatorname{sign}^{7}$ indicating an FFT in the empty space of the ICA. In this case, carotid ultrasonography showed severe stenosis, but the thrombus floating in the visible area could not be detected. Common treatments include medical therapy, resection, and endovascular therapy.

In a case report of 145 FFTs and a review of the literature, Bhatti et al. ${ }^{1}$ reported complete resolution of thrombi without cerebral infarction in 24 of 28 patients (86\%) treated with medical therapy. In addition, there was no difference in the outcome between medical and surgical treatments (including endovascular treatment). ${ }^{1}$ Medical treatment with anticoagulation is usually performed, and resection or endovascular treatment is often considered in cases that are resistant to medical treatment. However, in some cases, the thrombus becomes brittle and embolizes distally after anticoagulation; consequently, it is necessary to consider the treatment method in each case. ${ }^{8}$ In a case report series of 6 patients with FFT, Tolaymat et al. ${ }^{9}$ reported that carotid endarterectomy (CEA) was performed without neurological complications. In addition, in a case report series of urgent CEA and medical therapy (including standby CEA), all 14 patients treated with medical therapy had a good prognosis, but 4 of 15 patients who underwent urgent CEA had neurodegenerative findings due to new cerebral infarction. ${ }^{10}$

No study has yet compared resection with endovascular treatment. As with normal ICA stenosis, factors conferring high risk for CEA, plaque normalization of the stenosis, degree of calcification, stenosis elevation, the possibility of late rehabilitative intervention after CEA, use of thrombolytic and antithrombotic therapy, and the surgeon's proficiency should be considered. Here, we performed thrombus retrieval therapy and revascularization because of the onset of symptoms under antiplatelet medication, the possibility of unstable thrombus release and further distal embolization, the concern regarding further thrombus growth due to residual stenosis, and being able to treat after digital subtraction angiography. There have been reports of endovascular treatment of FFT with mechanical thrombectomy (MT) and CAS. In the absence of carotid stenosis with FFT, use of a stent retriever (SR) or a direct aspiration pass technique (ADAPT) with or without protection has been reported. ${ }^{4,11-13}$ In the case of stenosis, CAS alone or CAS and MT were performed using a protective device to prevent FFT migration, and many cases were treated without causing migration. The reports are summarized in Table 1. Reports of endovascular treatment of FFTs with stenosis can be broadly divided into 2 categories: (1) CAS under protection and (2) CAS followed by thrombus retrieval under protection.

TABLE 1. Summary of case reports and case series of endovascular treatment of carotid artery stenosis with FFT

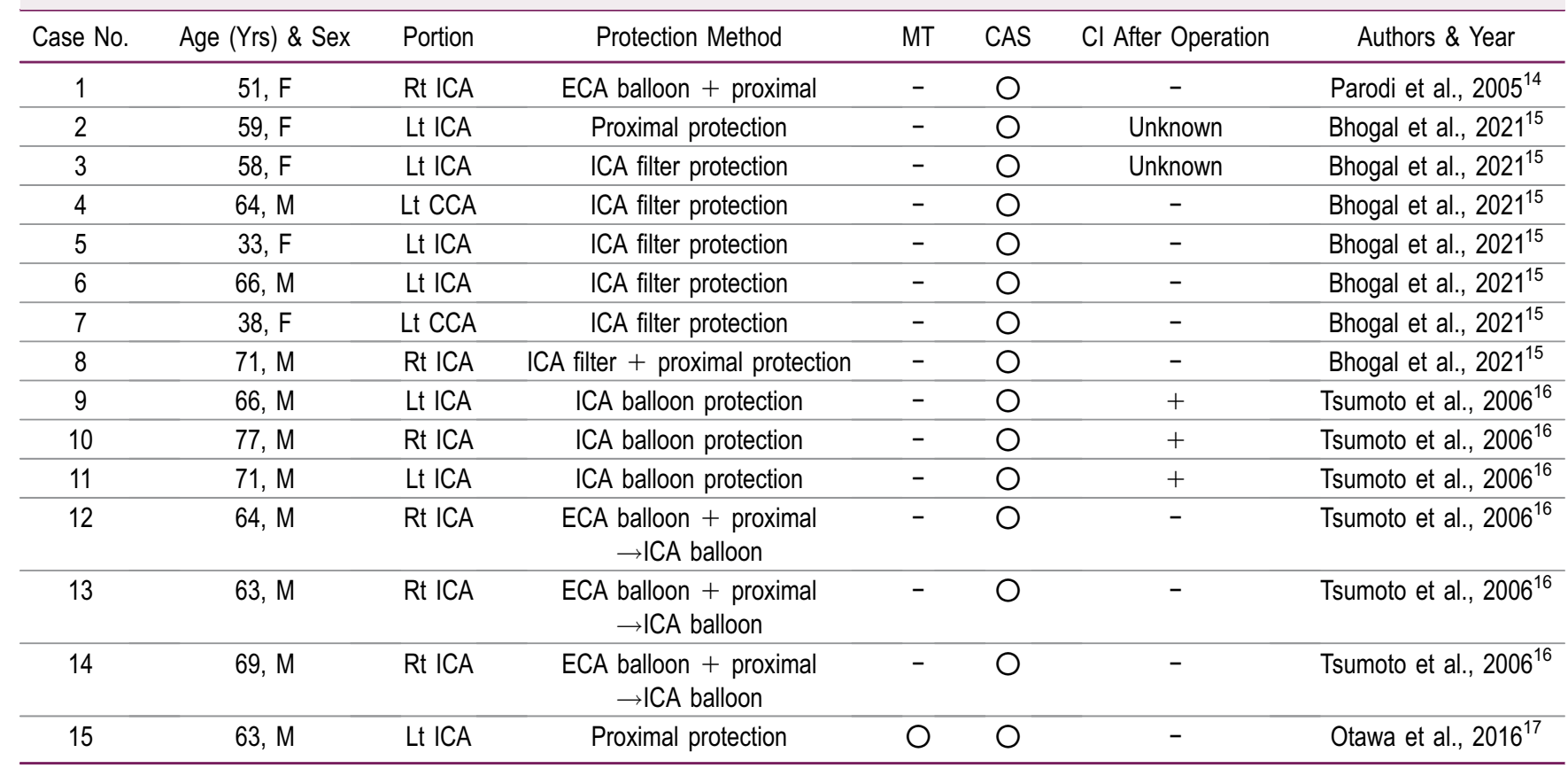

$\mathrm{Cl}=$ cerebral infarction. 
In the previous report summarized here, postoperative cerebral infarction was observed in 3 of 14 patients with CAS alone, and no migration was observed in the report of CAS combined with MT. However, there are only a few reports on the latter, and the results cannot be compared. However, considering the high risk of migration due to the instability of the thrombus, it may be advantageous to perform thrombus retrieval first. In addition, in CAS alone, the thrombus is crimped to the vessel wall by the stent. Considering the risk of thrombus fragmentation, distal embolization, and the possibility that the thrombus may not be completely covered by the stent, it is safer to perform thrombus retrieval first in that the thrombus can then be removed from the body. On the one hand, although we found no reports in the literature within the scope of our search, considering the risk of in-stent thrombus occlusion due to stenting in the acute phase, only thrombus retrieval in the acute phase and CAS in the chronic phase should be considered. On the other hand, it is difficult to decide whether thrombus retrieval should be performed using an SR or an aspiration catheter. In this case, we could retrieve the thrombus only with an aspiration catheter, which had the largest diameter at the time. The disadvantage of the suction catheter is that the suction catheter itself is thick, and therefore pre-PTA is required to insert the suction catheter, as in this case. In addition, if the clot is large, it may be difficult to recover it using the suction catheter alone in some cases.

For the use of an SR, as long as a small microcatheter can be guided distal to the stenosis, the SR can be deployed without treatment of the stenosis, but there is a great possibility that a thrombus in the stenotic area may be missed during stent removal. In the future, there will be cases in which a combination of an SR and a suction catheter will be necessary. In this case, we performed prePTA, thrombus retrieval by an aspiration catheter, and CAS to reliably remove the embolic source and treat the stenotic lesion presumed to be the cause of embolization. In addition, to minimize the risk of distal embolization and to allow immediate treatment in case of distal embolization, a guiding catheter with a balloon was placed in the CCA, PTA was performed at the stenosis to allow the passage of a large-bore aspiration catheter under flow reversal, and ADAPT was performed, followed by CAS. There is a previous report of FFT with right ICA stenosis in which ADAPT was performed with proximal protection of the CCA under echocardiographic guidance, followed by $\mathrm{CAS} .{ }^{17}$ In this case, we blocked the blood flow between the ECA and CCA to prevent an embolism caused by blood flow from the ECA collateral circulation. We believe that this procedure was useful to perform MT and CAS smoothly in a short time after blocking the carotid artery. Although we considered treating the patient under distal balloon protection, we performed thrombus aspiration under ECA and proximal protection because we believed that using an aspiration catheter under reversal flow from the ICA would increase aspiration efficiency. However, a suction catheter thicker than ACE68 cannot be placed in a guiding catheter with a 9-French balloon. Therefore, when using a suction catheter while blocking the ECA and CCA, it should be noted that the GuardWire for ECA occlusion should be guided from another guiding catheter.

\section{Lessons}

The prevalence of FFT is low, but there is a high possibility that FFT will become symptomatic and will need to be treated properly. PTA and thrombus aspiration and CAS under reversal flow may be effective treatments for FFT caused by ICA stenosis.

\section{Acknowledgments}

This work was supported by the Clinical Research Institute, National Hospital Organization, Kyusyu Medical Center.

\section{References}

1. Bhatti AF, Leon LR Jr, Labropoulos N, et al. Free-floating thrombus of the carotid artery: literature review and case reports. $J$ Vasc Surg. 2007;45(1):199-205.

2. Barnett HJM, Taylor DW, Haynes RB, et al. Beneficial effect of carotid endarterectomy in symptomatic patients with high-grade carotid stenosis. N Engl J Med. 1991;325(7):445-453.

3. Roy M, Roy AK, DeSanto JR, Abdelsalam M. Free floating thrombus in carotid artery in a patient with recurrent strokes. Case Rep Med. 2017;2017:4932567.

4. Carr K, Tew D, Becerra L, Siddall K, Dubensky L, Serulle Y. Endovascular aspiration of a symptomatic free-floating common carotid artery thrombus. Neuroradiology. 2018;60(10):1103-1107.

5. Sallustio F, Di Legge S, Marziali S, Ippoliti A, Stanzione P. Floating carotid thrombus treated by intravenous heparin and endarterectomy. J Vasc Surg. 2011;53(2):489-491.

6. Mees B, Robinson D, Fell G, Chu P. Symptomatic free-floating carotid thrombus extending to the skull base. Neurol Clin Pract 2014;4(6):536-537.

7. Menon BK, Singh J, Al-Khataami A, Demchuk AM, Goyal M. The donut sign on CT angiography: an indicator of reversible intraluminal carotid thrombus? Neuroradiology. 2010;52(11):1055-1056.

8. Watanabe M, Mori T, Imai K, Izumoto H, Hirano T, Uchino M. Distal migration of a floating carotid thrombus in a patient using oral contraceptives: a case report. J Med Case Rep. 2009;3:8389.

9. Tolaymat $\mathrm{B}$, Irizarry $\mathrm{K}$, Reif $\mathrm{M}$, et al. Considerations beyond stenosis for carotid endarterectomy in treating free-floating thrombus of the carotid artery. Ann Vasc Surg. 2019;60:221-228.

10. Buchan A, Gates P, Pelz D, Barnett HJ. Intraluminal thrombus in the cerebral circulation. Implications for surgical management. Stroke. 1988;19(6):681-687.

11. Fitzpatrick N, Motyer R, Gibney B, et al. Expanding the role of stent-retriever endovascular thrombectomy: a case series of freefloating thrombus. J Neurointerv Surg. 2018;10(12):1164-1167.

12. Giragani S, Balani A, Agrawal V. Stentriever thrombectomy with distal protection device for carotid free floating thrombus: a technical case report. J Neurointerv Surg. 2017;9(8):e33.

13. Yamaoka A, Miyata K, lihoshi S, Mikuni N. Endovascular treatment of asymptomatic free-floating thrombus in the carotid artery bifurcation: a direct aspiration first-pass technique under double balloon protection. BMJ Case Rep. 2019;12(8):e230295.

14. Parodi JC, Rubin BG, Azizzadeh A, Bartoli M, Sicard GA. Endovascular treatment of an internal carotid artery thrombus using reversal of flow: a case report. J Vasc Surg. 2005;41(1):146-150.

15. Bhogal P, AlMatter M, Aguilar Pérez M, Bäzner $H$, Henkes $H$, Hellstern V. Carotid stenting as definitive treatment for free floating thrombus-review of 7 cases. Clin Neuroradiol. 2021;31(2): 449-455.

16. Tsumoto $T$, Terada $T$, Tsuura M, et al. Carotid artery stenting for stenosis with intraluminal thrombus. Neuroradiology. 2006;48(1): 54-59.

17. Otawa M, Kinkori T, Watanabe K, Ando R, Tambara M, Arima T. A case of carotid free-floating thrombus treated by carotid ultrasonography-guided endovascular approach. Article in Japanese. No Shinkei Geka. 2016;44(6):489-494.

\section{Disclosures}

The authors report no conflict of interest concerning the materials or methods used in this study or the findings specified in this paper. 


\section{Author Contributions}

Conception and design: Tomoyose. Acquisition of data: Tomoyose, Okada. Analysis and interpretation of data: Tomoyose, Hara. Drafting the article: Tomoyose, Tsumoto, Yasaka. Critically revising the article: Tomoyose, Tokunaga, Yasaka. Reviewed submitted version of manuscript: Tomoyose, Miyazaki, Yasaka, Okada. Approved the final version of the manuscript on behalf of all authors: Tomoyose. Statistical analysis: Tomoyose. Administrative/technical/material support:
Tomoyose, Tsumoto, Hara. Study supervision: Tomoyose, Hara, Yasaka.

\section{Correspondence}

Ryuta Tomoyose: Kokura Memorial Hospital, Kitakyushu, Japan. ryuuta1115jp@yahoo.co.jp. 